\title{
Editorial
}

\section{Progress of Particle Flow, Fluid/Solid Mechanics, and Heat Transfer in Advanced Gas/Water Nuclear Reactors}

\author{
Nan Gui, ${ }^{1}$ Xiangdong $\mathrm{Li},{ }^{2}$ and $\mathrm{Xin} \mathrm{Tu}^{3}$ \\ ${ }^{1}$ Institute of Nuclear and New Energy Technology, Tsinghua University, Beijing, China \\ ${ }^{2}$ School of Engineering, RMIT University, Melbourne, VIC, Australia \\ ${ }^{3}$ University of Liverpool, Liverpool, $U K$ \\ Correspondence should be addressed to Nan Gui; guinan@mail.tsinghua.edu.cn
}

Received 14 November 2016; Accepted 15 November 2016

Copyright (C) 2016 Nan Gui et al. This is an open access article distributed under the Creative Commons Attribution License, which permits unrestricted use, distribution, and reproduction in any medium, provided the original work is properly cited.

Particle flow, fluid/solid mechanics, and heat transfer are very important to many technologies and applications of the advanced gas/water nuclear reactors. To better understand the relevant knowledge of particle flow, complex interactions (including multiphase thermal hydraulics), flow mechanics, and heat transfer certainly help improve the development of advanced nuclear reactors.

An introductory review of the accepted papers is presented here. In the paper entitled "A Calculation Method for the Sloshing Impact Pressure Imposed on the Roof of a Passive Water Storage Tank of AP 1000," to estimate the impact pressure imposed on the roof of water storage tank of AP 1000, a simplified method was proposed to approximate the difference between maximum wave height and roof height as the impact pressure head. By experimental validation, this method may overpredict the impact pressure. But the error is no more than $35 \%$. In the paper entitled "Thermal Analysis for the Dense Granular Target of CIADS," a new concept of a dense granular spallation target is proposed for which the tungsten granules are chosen as the target material for the China Initiative Accelerator Driven Reactor System (CIADS). After being bombarded by the accelerated protons from the accelerator, the tungsten granules with high temperature flow out of the subcritical reactor and the heat is removed by the heat exchanger. In the paper entitled "A Study on the Instantaneous Turbulent Flow Field in a 90-Degree Elbow Pipe with Circular Section," large eddy simulation was adopted to predict instantaneous flow field in the 90-degree elbow pipe. Instantaneous pressure information and velocity field were analyzed at specific monitoring points. In the paper entitled "Wave Characteristics of Falling Film on Inclination Plate at Moderate Reynolds Number," falling water film on an inclined plane is studied by shadowgraphy with different inclination angle and the film Reynolds number. Three distinctive regimes and three characteristic parameters of two-dimensional solitary wave pattern are examined, which are significant in engineering applications for estimation of heat and mass transfer on film flow. In the paper entitled "Parametric Investigation and Thermoeconomic Optimization of a Combined Cycle for Recovering the Waste Heat from Nuclear Closed Brayton Cycle," a combined cycle that combines an AWM cycle with a nuclear closed Brayton cycle is proposed to recover the waste heat rejected from the precooler of a nuclear closed Brayton cycle. The effects of several important parameters are investigated and the combined cycle performance is optimized based on a multiobjective function. In the paper entitled "Numerical Investigation on Bubble Growth and Sliding Process of Subcooled Flow Boiling in Narrow Rectangular Channel," a boiling phase change model in subcooled flow boiling is proposed, and VOF model combined with phase change model is adopted to simulate the single bubble growth and movement. Finally, in the paper entitled "Assessment of Prediction Capabilities of COCOSYS and CFX Code for Simplified Containment," the actual capability of CFX tool and COCOSYS code is assessed in prototypical geometries for simplified physical process-plume under adiabatic and convection boundary condition, respectively. 


\section{Acknowledgments}

We would like to thank all the contributors of this special issue for their support and cooperation. The evaluation of paper's quality by qualified reviewers is greatly appreciated as well. The work of Nan Gui was supported under the National Natural Science Foundation of China (Grant no. 51576211).

Nan Gui Xiangdong $L i$ $\mathrm{Xin} \mathrm{Tu}$ 

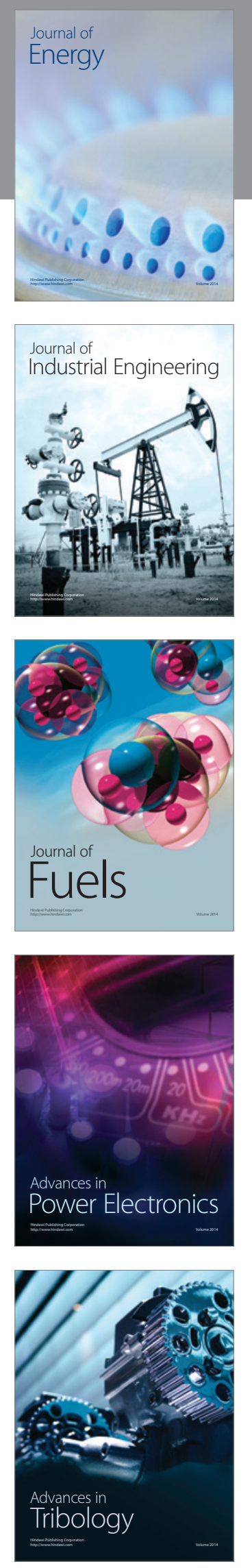
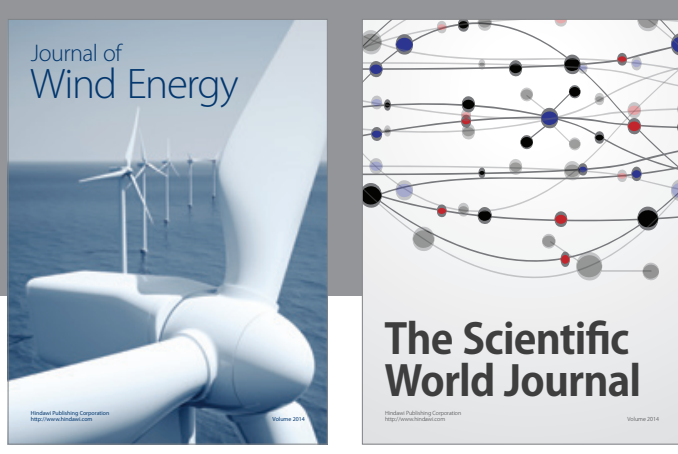

The Scientific World Journal
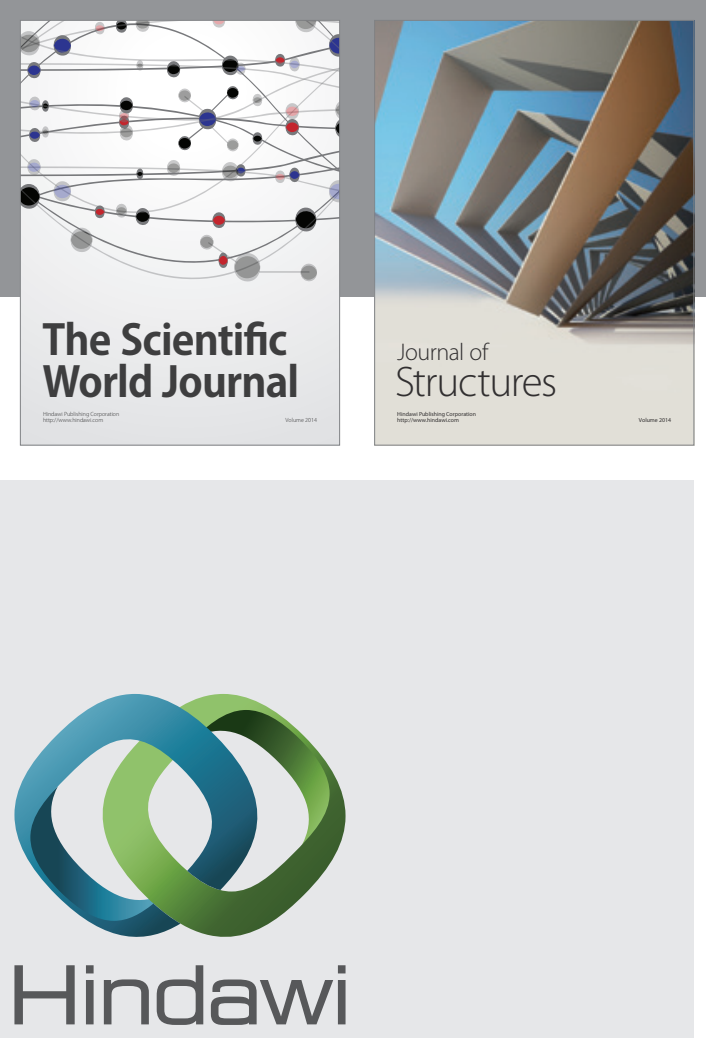

Submit your manuscripts at

http://www.hindawi.com
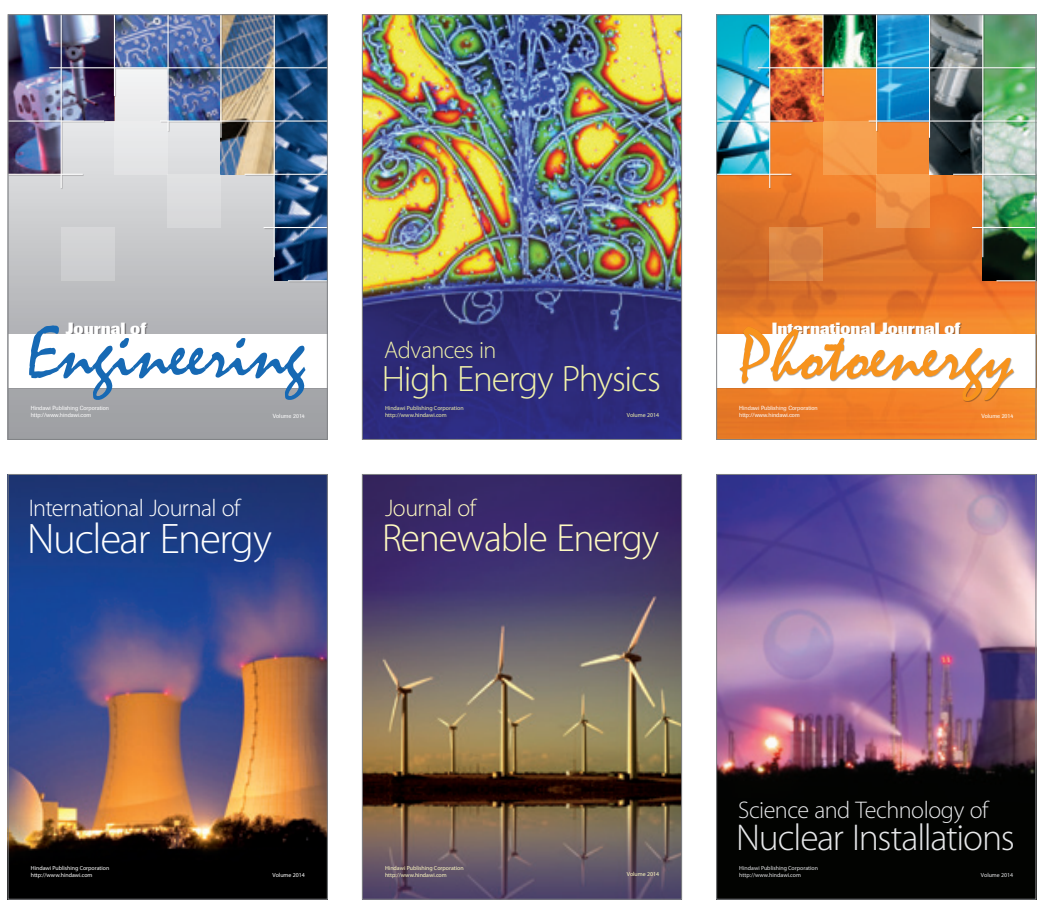
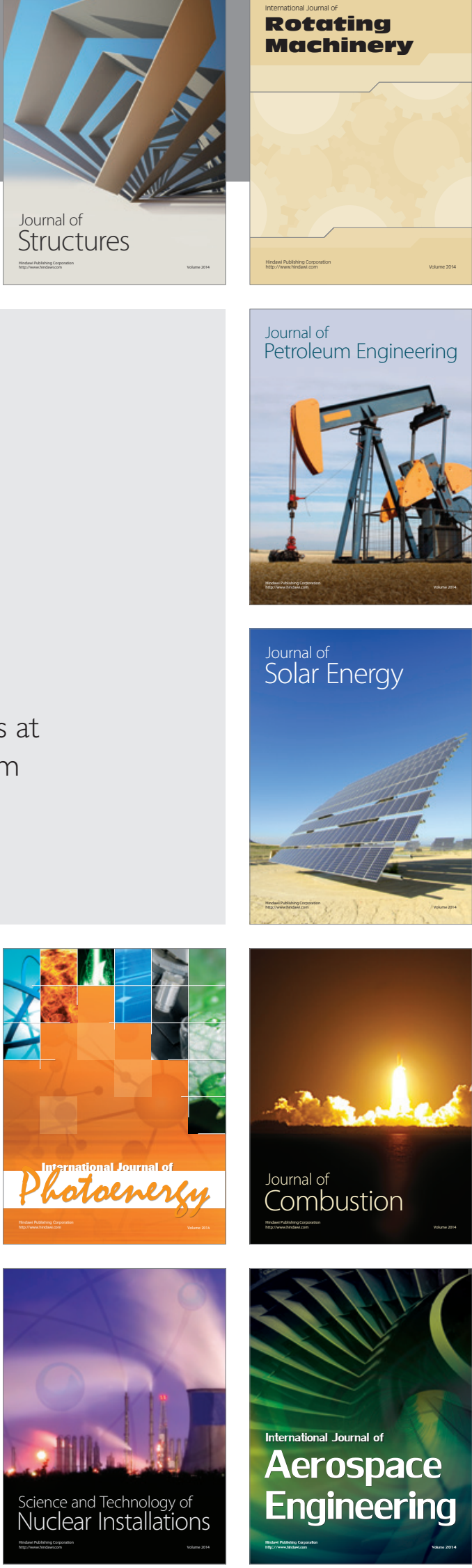